\title{
EDITORIAL
}

\section{Does diabetes therapy influence the risk of cancer?}

\author{
U. Smith • E. A. M. Gale
}

Published online: 14 July 2009

(C) Springer-Verlag 2009

Keywords Cancer - Insulin analogues - Insulin therapy · Metformin · Type 2 diabetes

\begin{abstract}
Abbreviations
AMPK AMP-activated protein kinase

FDA Food and Drug Administration

HMEC Human mammary epithelial cell

MAPK Mitogen-activated protein kinase

mTOR Mammalian target of rapamycin

NPH Neutral protamine Hagedorn

THIN The Health Information Network
\end{abstract}

\section{Introduction}

Type 2 diabetes is associated with three of the five leading causes of cancer mortality in the USA - carcinoma of the colon, pancreas and breast (postmenopausal) [1]. The excess risk for each cancer is $\sim 30 \%$ (colon), $\sim 50 \%$ (pancreas) and $\sim 20 \%$ (breast) [2-4]. Type 1 diabetes carries an excess cancer risk of $\sim 20 \%$, but involves a different range of tumours [5]. The major cancers linked with type 2 diabetes are also associated with obesity or insulin resistance, suggesting that factors other than glucose play

\footnotetext{
U. Smith

The Lundberg Laboratory for Diabetes Research, Department of Molecular and Clinical Medicine, Sahlgrenska University Hospital, Göteborg, Sweden

E. A. M. Gale $(\square)$

Diabetes and Metabolism, Medical School Unit,

Southmead Hospital,

Bristol BS10 5NB, UK

e-mail: Edwin.Gale@bristol.ac.uk
}

an important role [6]. These observations, although wellattested, have attracted relatively little interest within the world of diabetes. This is partly due to the dominant role of cardiovascular disease, which largely accounts for the twofold increase in mortality associated with type 2 diabetes [7], and partly, perhaps, because cancer has seemed unavoidable.

The latter assumption can no longer be considered correct, for several studies have shown metformin to be associated with a lower risk of cancer than insulin or sulfonylureas [8-10]. Bowker and colleagues examined the relationship between diabetes treatment and mortality in a health database from Saskatchewan, and found that cancer mortality was almost doubled among insulin users (HR 1.9, 95\% CI 1.5-2.4, $p<0.0001)$ relative to metformin users, and that sulfonylureas were also associated with increased mortality (HR 1.3, 95\% CI 1.1-1.6, $p=0.012$ ) [9]. A study published in this issue of Diabetologia confirms these observations, while showing that cancer risk in metformintreated patients is similar to that in patients who have not as yet received medication for diabetes. Furthermore, the paper suggests that the effect of metformin may be tumour-specific, in that its use was associated with a reduced risk of cancer of the colon and pancreas, but not of cancer of the breast or prostate [10].

The antitumour effect of metformin seems to be mediated via its ability to increase the AMP-activated protein kinase (AMPK) signalling pathway [11]. AMPK, which is activated by a rise in the AMP:ATP ratio, plays a key role in cellular energy balance. Activation restores levels of ATP by switching on ATP-generating pathways and switching off ATP-consuming pathways, and this enzyme is thought to mediate many of the metabolic actions of metformin [12]. Increased AMPK activity also leads to an inhibition of the downstream mammalian target of rapamycin (mTOR) complex; mTOR kinase integrates 
various cellular signals from growth factors, nutrition and energy state to regulate protein synthesis and cell growth. Rapamycin, the inhibitor of mTOR, and its derivatives have been tested in several cancer trials with some success. A study of human prostate cancer cells demonstrated a strong anti-proliferative effect of metformin [13]. This effect was unaffected by inhibition of the AMPK pathway, but was associated with cell cycle arrest in $\mathrm{G}_{0} / \mathrm{G}_{1}$ phase, together with a major reduction in cyclin D1 levels.

Another interesting mechanism for the anti-oncogenic effect of metformin has been postulated, based on the findings of a study of mice with $\mathrm{CD}^{+} \mathrm{T}$ lymphocytes which lack tumour necrosis factor receptor-associated factor 6 (TRAF6) and are unable to generate T memory cells [14]. This failure was associated with defective fatty acid oxidation. Metformin restored both the metabolic defect and generation of memory $\mathrm{T}$ cells. A further experiment showed that metformin treatment increased $\mathrm{CD}^{+} \mathrm{T}$ memory cell populations in wild-type mice, and enhanced the efficacy of anti-cancer vaccination. These intriguing findings indicate a shared mitochondrial nexus for metabolic and immune pathways, and imply that metformin may also have a direct influence upon immune competence [14].

The welcome news, therefore, is that metformin use is associated with a lower risk of some types of cancer, and may even find a role in the management of cancer in non-diabetic individuals. This does not alter the fact the type 2 diabetes is associated with an excess cancer risk, and that diabetes therapies that increase levels of circulating insulin might potentially contribute to this risk. More specifically, there is concern that high insulin levels and associated changes in the IGF-1 axis may accelerate the progression of existing cancer foci. Insulin treatment of type 2 diabetes was, for example, associated with a twofold increase in the risk of colorectal cancer, compared with other therapies, in an analysis that adjusted for prior use of metformin or sulfonylureas [15]. The same analysis reported that cancer risk increased by an estimated $20 \%$ for each year of insulin therapy. The paper by Currie et al. in this issue of the journal confirms an association between insulin therapy and colon cancer, and suggests that sulfonylureas may also carry an equivalent risk [10].

\section{Insulin and cancer}

The possibility of an association between insulin and cancer has attracted intense research interest among cancer epidemiologists, since cancers of the colon, breast and pancreas have all been associated with increased circulating levels of endogenous insulin in the non-diabetic population $[6,16]$. This might explain some of the overlap between cancer risk in diabetes, obesity and other conditions associated with insulin resistance [6]. There is a possible mechanistic basis for these epidemiological findings, in that insulin is a growth factor for a number of epithelial tumours in cell culture systems, and hyperinsulinaemia also produces a secondary increase in the availability of IGF-1another known tumour growth factor-which is mediated by a reduction in IGF binding protein-1 levels (IGFBP-1). These changes in the insulin-IGF-1 axis might be expected to favour the survival and progression of early malignant foci $[6,17]$. Tumour cells must overcome no fewer than six roadblocks to progression before a malignant growth can become established. These are the acquisition of selfsufficiency in growth signals, insensitivity to growthinhibitory signals, evasion of programmed cell death (apoptosis), limitless replication potential, sustained angiogenesis, and loss of barriers to tissue invasion [18]. Changes in the insulin-IGF-1 axis have the potential to support selfsufficiency in growth signals and resistance to apoptosis, and may thus offer an adaptive advantage to cancer foci struggling to survive in a hostile environment [17].

\section{The insulin-IGF-1 axis}

Insulin and IGF-1 are sister molecules that share a common ancestry but diverged early in vertebrate evolution, since when they have co-evolved with their receptors to subserve distinct metabolic or trophic functions. The insulin and IGF-1 receptors are tetrameric members of the receptor tyrosine kinase family that are composed of two extracellular $\alpha$ domains and two intracellular $\beta$ domains and share considerable sequence homology. The metabolic consequences of receptor stimulation are mediated by the phosphorylation of IRS proteins and activation of the phosphatidylinositol 3kinase-Akt/protein kinase B pathway (the Akt pathway); activation of this pathway promotes the familiar metabolic effects of insulin, although the Akt pathway may also transmit growth signals. The growth-promoting consequences of receptor stimulation are more generally mediated by the Ras-mitogen-activated protein kinase (MAPK) pathway (the Ras pathway), which promotes cell growth and differentiation by regulation of gene expression [19].

Readers unfamiliar with this system might assume that signal specificity is intrinsic to the molecular interaction of these receptors and their ligands, as predicted by the traditional lock-and-key model. The reality is more complicated, for in certain contexts the insulin receptor can transmit mitogenic signals and the IGF-1 receptor can transmit metabolic signals. This is because the insulin-IGF1 axis functions within a dense and highly flexible signalling network, and differences in signalling specificity may vary with the target tissue, the density and spatial localisation of receptors, the kinetics of ligand binding, and downstream modulation of post-receptor signalling, all of 
which contribute to this remarkable functional plasticity, quite apart from any variation in the ligand itself [20].

A whole new dimension is superimposed upon this preexisting complexity when a cell undergoes malignant transformation, for-consistent with the dictum that 'oncology recapitulates ontogeny'-tumour cells can reacquire signalling capabilities that are otherwise only seen in the early stages of development. These include a variant form of the insulin receptor known as IR-A, which is abundantly expressed in both fetal and cancer tissues and is responsive to IGF-2 as well as to insulin [21]. Cancer cells may thus overexpress not only the insulin and IGF-1 receptors, but also IR-A and hybrid receptors formed by recombination of their constituent proteins.

In summary, tumour cells equipped with this aberrant signalling capacity may become dependent upon the trophic effects of insulin and/or IGF-1, thus accelerating their own growth and acquiring increased resistance to apoptosis [22]. Overexpression of this network of proteins is commonly observed in cancers of the colon, breast, pancreas and prostate. It should, however, be appreciated that cancers arise from multiple fortuitous mutations, and are therefore heterogeneous in the extreme. Some in vitro cancer cell lines are much more responsive to insulin and IGF-1 signalling than others. The reported effects of insulin on tumour cell lines are highly variable, as we will shortly see, and differences between cell lines, experimental conditions and concentrations of insulin must be taken into account when interpreting results from such reports. This having been said, there is abundant evidence that insulin can, in some circumstances, promote the growth of both healthy and malignant cell lines in culture systems.

Cancers take many years to develop, and it is therefore surprising that studies such as those reported in this issue of Diabetologia [10, 23-25] can claim to detect differences in cancer rates within a few years of exposure to different therapeutic agents. These observations, if confirmed, strongly suggest that the effects we are witnessing arise from differences in the rate of development of pre-existing malignant foci rather than malignant transformation and new cancer cell formation. This inference is consistent with the observed effects of insulin on cells in culture. Further support for this view comes from autopsy studies showing that a high proportion of people in an ageing population harbour early cancers. Prostate cancers, for example, are present in some $50 \%$ of 70 -year-old men and in $80 \%$ of those over the age of 90 [17].

\section{Insulin analogues and cancer}

On 9th April 2008, Pfizer (New York, NY, USA) issued a 'Dear Healthcare Professional' letter to the effect that inhaled recombinant human insulin had been associated with six new cases of lung cancer in clinical trials, with one further post-marketing report in a patient treated with Exubera. A single case had been reported in comparatortreated patients, and all cases had a history of prior cigarette smoking [26]. The company stated that this sixfold increase in risk $(0.13 / 1,000$ cases vs 0.02$)$ did not prove a causal connection, but the observation may have helped to motivate the precipitate removal of Exubera from the market on 17th October 2007 [27]. The effects of massive local concentrations of insulin in the lung cannot, however, be extrapolated to the safety or otherwise of subcutaneous insulin.

Three potentially relevant observations provide the essential background for any discussion about insulin therapy and cancer: (1) the epidemiological finding that insulin concentrations within the physiological or therapeutic range are associated with the rate of tumour diagnosis; (2) laboratory findings that the intrinsic mitogenicity of insulin may vary according to the functional plasticity of the insulin-IGF-1 signalling network, particularly in tumour cells; and (3) the demonstration that some tumour cell lines are responsive to changes in the ambient concentrations of either or both these ligands. Concerns that the insulin analogues might be associated with an increased risk of tumour progression [28, 29] must be appreciated against this complex background.

The introduction of biosynthetic human insulin opened the way for 'designer' insulins modified for faster or more sustained effects following injection, and it soon became apparent that some alterations of the insulin molecule increase its trophic effects, as demonstrated by accelerated DNA synthesis and cell division in cell culture systems, typically human mammary epithelial cells (HMECs). These effects are mediated by prolonged binding to the insulin receptor, or by increased cross-reactivity with the IGF-1 receptor [30,31], and all new insulins are routinely screened for their effects on cell growth in the course of preclinical evaluation. Insulin B10Asp, the first of the analogues to be developed, was based on a single amino acid substitution. This was, however, sufficient to produce a tenfold increase in mitogenicity, compared with human insulin. In the light of this observation, the regulatory authorities required 2 year carcinogenicity studies in rodents, as against the standard 1 year toxicity testing, and the insulin was withdrawn when mammary tumours appeared in rats [32].

Further experience revealed that the insulin and IGF-1 receptors recognise the terminal part of the insulin $\mathrm{B}$ chain and extensions into the $\mathrm{C}$ chain differently. Modification of B26-B30 regions of the B chain increases IGF-1 receptor binding, as does modification of the B10 residue and extension of the $\mathrm{B}$ chain by addition of arginine residues [33]. Changes at both sites have additive effects, in that 
AspB10DiArg insulin, used for experimental purposes only, produces a 90 -fold increase in binding to the IGF-1 receptor on HMECs. Insulin glargine (A21Gly,B31Arg,B32Arg human insulin) also contains arginine residues at positions B31 and B32, together with a glycine substitution at A21; insulin aspart (B28Asp human insulin) carries a B28Asp substitution, and in insulin lispro (B28Lys,B29Pro human insulin) the sequence of proline and lysine residues at B28/ B29 in human insulin are reversed. Insulin detemir, B29Lys ( $\varepsilon$-tetradecanoyl), desB30 human insulin carries a fatty acyl chain attached to the end of the B chain. The ability of analogues to stimulate HMEC growth generally correlates with their ability to bind to the IGF-1 receptor, but prolonged interaction with either receptor also appears necessary for stimulation of mitotic activity [30].

Kurtzhals and colleagues used a variety of systems, including human osteosarcoma cells, to compare receptor affinities and mitogenic potencies of the insulin analogues in current clinical use, and found that insulin glargine has a six- to eightfold increase in receptor affinity and mitogenic potency compared with human insulin [33]. Sanofi-aventis had previously observed a similar increase in mitogenic activity in osteosarcoma cells [28]. In contrast, the two short-acting analogues were reported to resemble human insulin in most respects other than a slight increase in IGF1 receptor affinity for insulin lispro. Insulin detemir had reduced metabolic and mitotic potencies in vitro compared with human insulin, presumably because it carries a fatty acyl chain, which might be expected to interfere with receptor binding, but is, for technical reasons, difficult to compare with other insulins in such systems [33].

As indicated in the previous section, insulin analogues have been tested in tumour cell lines with variable results. A pancreatic cancer cell line responded similarly to insulin glargine and human insulin, and survival of insulin glargine-treated patients following treatment for pancreatic cancer did not differ from that of patients on insulin or controls [34]. In another study, colorectal, breast and prostate cell lines showed proliferative changes and increased resistance to apoptosis in response to exposure to pharmacological doses of insulin glargine, insulin detemir and insulin lispro, but not to human insulin [35]. Another recent paper tested the effect of exposure to insulin analogues on a panel of neoplastic and non-neoplastic mammary epithelial cell lines. Preliminary screening for the insulin/IGF-1 receptors indicated that growth of the malignant cell line MCF7 was strongly promoted by insulin glargine, but not by other insulins, at dosage levels in the picomolar range, comparable to those found in the circulation of insulin-treated patients. This effect was strongly linked to activation of the IGF-1 receptor and the MAPK pathway. Other cell lines carrying insulin/IGF-1 receptors did not respond in this way [36].
Insulin glargine is partially degraded at the injection site, yielding two bioactive products known as M1, which lacks the diarginine residues at B31 and B32, and M2, which has additional deletion of the threonine at B30. Both products retain the glycine substitution for asparagine at A21. These are therefore closely similar to, but not identical with, human insulin $[37,38]$, and their mitogenicity appears to be low [38]. All three forms (unchanged insulin glargine, M1 and M2) enter the circulation. Further degradation of insulin glargine to M1 occurs on exposure to serum, probably mediated by carboxypeptidase enzymes [38]. These observations suggest that insulin glargine behaves to some extent as a prodrug, generating bioactive breakdown products both at the site of injection and within the circulation. It follows that insulin glargine may be less mitogenic in vivo than in vitro, but the studies suggest considerable inter-individual variation, and a substantial proportion of the insulin injected will, on present evidence, reach the cells in the form of unaltered glargine.

In summary, it is currently impossible to extrapolate from the in vitro to the in vivo situation with any confidence. The mandatory preclinical testing procedures to which all the insulin analogues in current clinical use have been subjected are therefore insufficient to confirm or to exclude a possible cancer risk in humans. Preclinical testing has, however, identified legitimate cause for concern regarding some of the analogues.

\section{Carcinogenicity studies in rodents}

Preclinical evaluation of the analogues includes safety tests in animals, and development of insulin B10Asp was halted when this was shown to promote the development of mammary tumours in female Sprague-Dawley rats [32]. The daily doses tested were 12.5, 50 and $200 \mathrm{U} / \mathrm{kg}$, and malignant tumours developed in $0,11 \%$ and $23 \%$ of the rats, respectively. Insulin glargine, by contrast, was tested at the lower daily doses of 2, 5 and $12.5 \mathrm{U} / \mathrm{kg}$ [39], the last of which is said to correspond to human daily doses of approximately $100 \mathrm{U}$ (rats) or $50 \mathrm{U}$ (mice). It is worthy of note that insulin B10Asp would have passed the carcinogenicity testing to which insulin glargine was subjected and would now be in clinical use. Interpretation of the insulin glargine studies was further complicated by a very high early mortality rate, which was probably due to hypoglycaemia at the higher insulin doses. This led the US Food and Drug Administration (FDA) to comment that 'the findings in female mice were not conclusive due to excessive mortality in all dose groups' [40], a caution which, curiously, finds no echo in the published report of the study [39]. Mammary tumours did indeed develop in $10-20 \%$ of the female rats, but were no more common in 
rats treated with insulin glargine than in those treated with neutral protamine Hagedorn (NPH) insulin or control solutions.

In summary, and despite the rapid increases in knowledge that have accrued since the insulin analogues reached the market, we can make no firm judgement as to whether the insulin analogues do or do not enhance cancer risk on the basis of preclinical or laboratory testing. Since prospective clinical trials are evidently impracticable (who would agree to participate?), this possibility can only be addressed by observational studies in humans.

\section{First observations in man}

A large observational study submitted to this journal last year suggested that use of insulin glargine is, after adjustment for dose, associated with a possible increase in tumour risk in humans [23]. Interpretation of this analysis proved controversial, but the implications were serious. A special advisory group, convened by the EASD, agreed that it would be premature to publish these findings in isolation, and that replication was needed. The three other observational analyses presented in this issue of Diabetologia were therefore commissioned to examine the safety of this insulin $[10,24,25]$, and the main findings will be summarised here. Coincidentally, a further paper in this issue reports a prospective evaluation of the risk of retinopathy progression in patients treated with insulin glargine or human NPH insulin [41]. Additional safety data relating to cancer risk in this and other studies have been made available to our journal and will be published shortly.

German insurance study In this report [23], which triggered the remainder, Hemkens and colleagues present data from a large insurance dataset, and compare the rate of diagnosis of malignant tumours in patients treated with human insulin, as against three of its analogues: insulin lispro, insulin aspart and insulin glargine. Insulin detemir, more recently introduced to the German market, was not included. The 127,031 patients (39\% of all those on insulin) in this large population sample had all started insulin treatment since 2000, and were all treated exclusively with human insulin (soluble and/or $\mathrm{NPH}$ ) or one of the three analogues. Of these, 95,804 (75.4\%) were exclusively on human insulin, 23,855 (18.8\%) were on insulin glargine alone, 3,269 (2.6\%) were on insulin lispro and 4,103 (3.2\%) were on aspart alone. It should be noted that, in Germany, patients with type 2 diabetes are often treated with preprandial doses of rapid-acting insulin without a basal supplement. The insulin dose was extracted from the medical records. Although classification of diabetes is not specified in the register, those included in this analysis will almost all have had type 2 diabetes, an interpretation supported by the median age of $\sim 67$ years in all four groups.

The major finding of this analysis was a strong correlation between insulin dose and cancer risk, regardless of insulin type. The influence of dose greatly complicated the analysis, since the crude incidence of malignant neoplasms was higher in patients on human insulin than in those receiving one of the three analogues, but patients on human insulin were also treated with larger doses of insulin. Insulin glargine users were prescribed a median of $\sim 22 \mathrm{U} /$ day (95\% quantile $\sim 59 \mathrm{U}$ ), compared with a median of $\sim 37 \mathrm{U}(95 \%$ quantile $\sim 100 \mathrm{U})$ for human insulin. Insulin glargine thus carried a significantly lower risk of cancer than human insulin in the unadjusted analysis, but the risk ratio reversed itself when insulin dose was allowed for, such that the rate of diagnosis of cancer and all-cause mortality both rose more steeply at higher doses of insulin glargine relative to human insulin. The adjusted HR for diagnosis of a cancer was 1.31 (95\% CI 1.20-1.42) for individuals on $50 \mathrm{U}$ of insulin glargine daily, as against $50 \mathrm{U}$ of human insulin. Dose for dose, insulin glargine thus appeared to carry a higher risk of cancer than human insulin.

As might be imagined from the somewhat complex nature of the analysis, this report created a dilemma for the journal. Our referees expressed a number of major reservations. These ranged from biological implausibility, given the short median period (1.31 years for insulin glargine) of exposure to each of the insulins, to the limited overlap between the dose ranges, the unexplained effect of insulin glargine on all-cause mortality, the lack of overall difference in cancer risk between the four insulins in the crude analysis, failure to correct for BMI in the doseresponse analysis, and a number of technical considerations. Nor was it possible to break the findings down according to the nature of the tumour - a major limitation given the low probability that any one agent might produce a non-specific increase in all types of cancer. Three of the six referees initially recommended rejection on the basis of these limitations, some of which were inescapable. On the other hand, strengths of the study included its large size, and its main findings survived a searching and prolonged review process. We anticipate that it will continue to generate controversy following publication, and concluded that it would be premature to publish these hypothesisgenerating data in isolation.

Publication was therefore made conditional upon the performance of additional studies, and these terms were accepted by the authors. Two national diabetes registries in Sweden and Scotland were therefore invited to run their data against those of their respective cancer registries [24, 25]. The overall null hypothesis was that patients treated with insulin analogues were not more likely to be diagnosed with cancer during the period of observation. 
At a later stage, a further analysis was commissioned from Pharmatelligence (Cardiff, UK), a commercial organisation with a well-characterised diabetes database previously obtained from The Health Information Network (THIN) in the UK [10].

Sweden The Swedish study linked data from a number of registries to identify 114,841 patients who received prescriptions for insulin in 2005 [24]. These records were then linked to data from the cancer registry for the two subsequent calendar years. The specific focus was on insulin glargine, as noted above, and some limitations should be noted. For example, duration of insulin therapy and exposure to other insulins prior to 2005 could not be considered. Patients were then divided into three groups: insulin glargine only (5,970 individuals), insulin glargine plus other insulins (20,316 individuals) and insulin users not on insulin glargine (88,555 individuals). Classification of diabetes was based on age at diagnosis, and those diagnosed after 30 years of age, including almost all those on insulin glargine alone, were considered to have type 2 diabetes. Insulin dose could only be estimated in terms of the number of insulin prescriptions filled, which meant that a dose-response relationship could not be examined in the same way as in the German study [23], which was based on recorded insulin doses. The endpoints were diagnosis of any neoplasm, and a diagnosis of a cancer of the breast, prostate or gastrointestinal tract. Joint consideration of all gastrointestinal tumours might be considered a further limitation of this study, since colon cancer is a much stronger candidate for an insulin effect.

The analysis found no statistically significant difference in cancer incidence between the two largest groups, those on insulins other than insulin glargine, and those on insulin glargine plus other insulins. Those on insulin glargine alone did, however, have a higher risk of breast cancer than those on insulins other than insulin glargine, with an RR of 1.99 (95\% CI 1.31-3.03), all other cancer risks being equal. This observation was robust in statistical terms, in that it was little affected by any of the subsequent adjustments that were made, and metformin use did not emerge as a confounder.

As in any observational study, this finding must be interpreted with caution. To begin with, the number of breast cancers was relatively low: 25 cases on insulin glargine vs 183 on insulins other than insulin glargine. Furthermore, it is puzzling that the reported effect should be limited to users of insulin glargine alone, rather than all insulin glargine users regardless of other insulins. The insulin glargine plus other insulin group did, however, contain a much higher proportion of younger patients (presumably on basal bolus therapy) than the other two groups. This indicates the strong possibility of an allocation bias, sometimes termed 'confounding by indication', i.e. differences between exclusive insulin glargine users and the comparator groups that might also influence their relative risk of breast cancer. Statistical corrections can limit this possibility, but cannot rule it out. Conversely, the observation has biological plausibility, for breast cancer would be a prime candidate for an insulin glargine effect in any a priori hypothesis based on laboratory data.

Scotland The Scottish analysis [25] is based on a national clinical database which covers almost everyone with diagnosed diabetes in Scotland. The analysis included all patients exposed to insulin therapy for the calendar years 2002, 2003 and 2004. An open cohort analysis included 49,197 patients on insulin, divided, as in the Swedish analysis, into insulin glargine alone, insulin glargine plus other insulins, and non-glargine insulins. These groups were then matched with cancer registry data validated up to the end of 2005. The analysis considered overall cancer incidence, and the frequency with which cancer of the breast, colon, prostate and pancreas were diagnosed. As in the Swedish study, there were clear differences between the patient groups; for example, those on insulin glargine alone were older than those on insulin glargine plus other insulins (68 vs 41 years) and users of other insulins (60 years). Not surprisingly, those on insulin glargine alone were also more overweight, more hypertensive, and more likely to be on oral glucose-lowering agents; $97 \%$ had a diagnosis of type 2 diabetes, as against $37 \%$ of those on insulin glargine plus other insulins. Relatively few Scottish patients were on insulin glargine at the time of study, with 3,512 on insulin glargine plus other insulins and only 447 on glargine alone. Those on insulin glargine with rapid-acting insulin had a slightly lower rate of cancer progression than did the human insulin group (HR $0.8,95 \%$ CI $0.55-1.17, p<0.26$ ), but those on insulin glargine alone had a higher overall rate (HR $1.55,95 \%$ CI 1.01-2.37, $p=0.045$ ). The number of site-specific cancers was small, but there were more cases of breast cancer in those on insulin glargine alone, compared with those on non-glargine insulins (HR 3.39, 95\% CI 1.46-7.85, $p=0.004)$. Although limited by sample size, this study also found that more cancers were diagnosed in those on insulin glargine alone. This observation was unaffected by statistical adjustments, but the authors conclude that their findings are more likely to have arisen from allocation bias than from a biological effect of insulin glargine.

UK GP database This analysis [10], based on records obtained from THIN, had the advantage of an established database with carefully defined sub-categories according to diabetes therapy. This database also enabled cancer risk to be determined in patients on monotherapy with metformin or sulfonylureas, on combination therapy with 
both, or on insulin (subdivided into insulin glargine only, NPH insulin only, human biphasic and analogue biphasic insulin only). An additional group of diet-treated diabetes, plus patients in the 3 year period prior to their diagnosis of diabetes, allowed cancer risk to be examined in medication-naive individuals. The analysis was limited to patients who entered a given treatment category later than the year 2000, although insulin users might, for example, have previously taken oral agents. This dataset was therefore more sharply defined in terms of diabetes therapy than the two national registries, but was also smaller.

The most striking finding to emerge from this analysis was the protective effect of metformin. This has been noted previously [9], but the present analysis has shown that the risk of cancer in metformin-treated patients is equivalent to that in treatment-naive individuals prior to diabetes therapy, and that the rate of cancer development associated with monotherapy with sulfonylureas or insulin is lower when these therapies are combined with metformin. Furthermore, metformin was associated with a reduced rate of cancer of the colon or pancreas, but no reduction was seen for cancer of the breast or prostate. The difference in risk of pancreatic cancer was striking, yet is consistent with experimental studies in hamsters [42]. It has also recently been shown that metformin abrogates sitagliptin-induced pancreatic ductal metaplasia, a precursor of carcinoma, in a rat model of type 2 diabetes [43]. These observations suggest that metformin may come to play a major role in cancer prevention in diabetes. For present purposes, however, the points to note are that concomitant metformin use is potentially a major confounder when it comes to estimating the risks of insulin therapy. Furthermore, the lack of effect of metformin on breast cancer, if confirmed, might help to explain why this particular cancer has tended to emerge from the analyses conducted in the previous two studies [10].

This study was essentially negative when it came to comparison of the four insulin-treated groups, whether in terms of all cancers or cancer of the breast, pancreas, or colorectal cancer, or a basket of all three cancer types. It will also be noted that the four insulin-treated groups were less evidently heterogeneous than patients in the other analyses we have considered. Numbers were, however, relatively small, with 2,286 on insulin glargine alone, compared with 1,262 on NPH insulin, and once again a dosage-based comparison, as performed in the German study [23], did not prove feasible.

\section{Insulin glargine and retinopathy}

A further safety concern requiring human studies arose when one of the early clinical trials [44] was reported to have observed a threefold increase in retinopathy progression with insulin glargine compared with human insulin [41]. Curiously, this fact is not mentioned in the original report [44], which concludes with the statement that insulin glargine has a safety profile that, apart from reduced nocturnal hypoglycaemia, is 'otherwise similar to NPH insulin'. Equally curious, a later review also cites the same paper as documenting a three step or greater retinopathy progression in $7.5 \%$ of those on insulin glargine vs $2.7 \%$ of those on NPH $(p<0.05)$ [45]. Since IGF-1 has a role in normal retinal vascular function and disease [46], this observation raised the possibility that insulin glargine might also have mitogenic effects on the vascular endothelium. The FDA required prospective comparative studies of retinopathy progression in patients taking human and glargine insulins in 1999 [28], and this work finally culminated in the report published in this issue of Diabetologia. Reassuringly, this analysis was entirely negative.

\section{What does it all mean?}

It will be evident from the foregoing that we have entered an area of considerable complexity. A number of useful conclusions may, however, be drawn, and questions can be formulated in terms that should permit an answer within a relatively short space of time.

To begin with, there is a school of thought that has maintained that there has been little or no case for insulin glargine to answer in terms of the laboratory and preclinical data. We do not accept this view. It is indeed difficult to extrapolate from preclinical testing to the clinical situation, as we have emphasised, but at least one of the analogues has demonstrated clear evidence of increased mitogenicity, and at concentrations similar to those achieved in clinical use. Although there have been negative studies, the growth of some tumour cell lines is clearly enhanced by insulin. There is indeed a case to answer.

Any consideration of the possible effect of the insulin analogues on cancer growth must allow for the fact that circulating levels of endogenous insulin appear to be associated with cancer risk in obesity and other insulinresistant conditions, including type 2 diabetes. Epidemiological association does not prove causation, but there are mechanistic data to support a direct role for insulin. This being the case, treatments that elevate circulating insulin levels in diabetes might potentially carry an increased risk of cancer. This possibility is strongly suggested by the powerful influence of insulin dose upon cancer risk shown in the German study [23], an effect seen with all types of insulin. This question requires further investigation, and the potential effect of the analogues, if any, must be judged in the light of this information. 
A further point to consider is that differences in cancer risk between treatments have emerged within a remarkably short period of exposure to the putative agent. This is almost without precedent in the field of cancer [24], and the observation, if confirmed, can only be interpreted in terms of activation or accelerated progression of latent malignant foci. The potential importance of factors that promote cancer progression is suggested by the observation that the rate of clinical prostate cancer is about tenfold greater in the USA than in Japan, whereas the prevalence of latent prostate cancer in autopsy studies is much the same in the two populations [17] (Fig. 1). Breast cancer screening has been implemented in both Sweden and Scotland, and might have contributed to earlier diagnosis. There is no reason to believe that insulin therapy causes cancer, but there is, nonetheless, reason to suspect that high concentrations of insulin may promote its development.

When it comes to interpretation of the studies themselves, it is important to note that the model upon which the German analysis is based depends on a number of

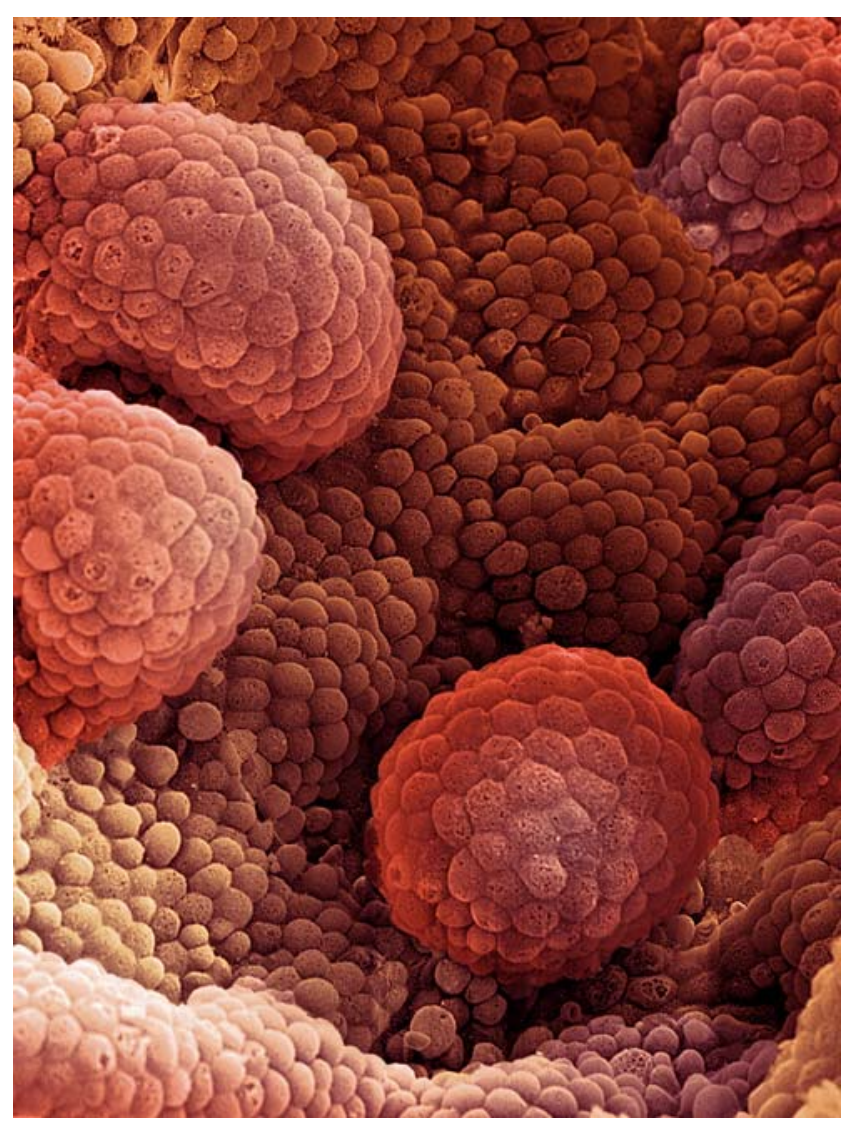

Fig. 1 Scanning electron micrograph showing prostate cancer. Prostate cancer appears to have a weak negative association with type 2 diabetes, possibly because a common polymorphism in the HNF1B gene, which predisposes to type 2 diabetes, also protects against prostate cancer [54]. Image from Steve Gschmeissner/Science Photo Library assumptions that may or may not prove to be justified. This study does, however, introduce the issue of dose, which may prove central to future consideration of this issue. The Swedish and Scottish studies [24, 25], which were especially commissioned to consider the safety of insulin glargine, are reasonably consistent. Both show a clear difference between the cancer risk of the group on insulin glargine plus other insulin and that of the group on insulin glargine alone. The demographic characteristics of both groups also differ, in that the former groups were composed largely of younger patients on basal bolus therapy. The baseline risk of cancer is much lower in this age group, together with the likelihood of pre-existing cancer foci. Furthermore, a proportion had type 1 diabetes, which is associated with a different range of cancers [5]. Statistical adjustment cannot fully compensate for biological differences between groups. The insulin glargine only groups also differed, although to a lesser extent, from the comparator groups on human insulin. The German study indicated an increased cancer risk in both sexes, suggesting that it would be premature to focus further attention on one specific tumour type to the exclusion of all others. This having been said, both studies independently indicate a signal for breast cancer, a biologically plausible tumour, and this observation cannot simply be ignored.

The THIN study managed to match the study groups more closely [10]; it also allowed a time-matched comparison to be made between monotherapy with human insulin and insulin glargine, which was not possible in the previous two studies $[24,25]$. In the event, no difference emerged between the therapies [10]. The number of patients studied was, however, relatively low, and interpretation of all three studies must allow for the relatively low frequency of breast cancer: 25 cases in patients on insulin glargine alone in Sweden, six cases in Scotland and ten cases in THIN. The latter study therefore provides some reassurance in relation to the two previous studies, but does not resolve the question at issue.

\section{Where next?}

The difficulties we encountered during the course of this analysis provide an excellent demonstration of the problems and pitfalls of observational studies [47, 48]. Prospective clinical trials set out to compare like with like, whereas pharmacoepidemiological studies such as those reported here almost inevitably need to make statistical adjustments for unmatched comparisons. You can only correct for confounders when you know what the confounders are. A prospective clinical trial would be the best way of resolving the issue, but would be unfeasible, arguably unethical, and 
far too slow to perform. Clinical trial data held on file by sanofi-aventis may allow some of these questions to be answered, but independent review would be an essential element of any such analysis. An additional approach, supplementary to the foregoing, would be a much larger pharmacoepidemiological analysis, jointly designed and conducted by industry and representatives of the scientific associations, and independently analysed. We believe that such a study would achieve as close an approximation to the truth as is likely to be possible.

\section{Summary and conclusions}

The focus of this series of investigations has been on insulin glargine. It has not proved possible to place insulin detemir under similar scrutiny, but it would be prudent for this insulin analogue to be investigated in more detail. On current evidence, the short-acting analogues do not appear to present a potential problem. With respect to insulin glargine, it is in no one's interest to mount a witch-hunt against this popular and widely used insulin-many will reflect upon the fate of rosiglitazone-but it is in everyone's interest for the truth to be known. The evidence presented in this set of papers is sufficient to establish that there is a case to answer, but is entirely insufficient to bring in a verdict. Certain reassurances do, however, seem justified. There is no evidence that insulin, however formulated, causes cancer. There is no evidence of an overall increase in the rate of cancer development in patients on insulin glargine, and some suggestion that the risk may actually be reduced. There is no evidence of harm in type 1 diabetes, or in premenopausal breast cancer. On the other hand, it has to be said that insulin glargine has not been shown to be more effective than human insulin in achieving glycaemic control in type 2 diabetes; its main benefit (if any) is in relation to symptomatic episodes of nocturnal hypoglycaemia [49-52]. We have safe and effective alternatives to offer our patients with type 2 diabetes.

The observations presented here require further analysis and evaluation, and are likely to open a much wider debate. Once the safety of the analogues has, as we would all wish, been confirmed, the wider debate will centre around the relationship between insulin and cancer, and the possibility of reducing this risk by lifestyle measures and metformin. One point has become abundantly clear, and this is that cancer must now be numbered among the complications of diabetes. Furthermore, and as with ischaemic heart disease, cancer is associated with a higher mortality in those with diabetes than in those without [53]. Cancer risk and prevention may become increasingly important considerations in diabetes therapy, and the implications of the studies reported here are likely to be very far-reaching.

Acknowledgements This article is dedicated to the memory of Michael Berger. We think he might have enjoyed reading it. Our grateful thanks are due to the teams of investigators in different countries who worked under high pressure to generate the data reported in the accompanying papers, and managed to do so in time for our joint publication deadline.

Duality of interest The authors declare that there is no duality of interest associated with this manuscript.

\section{References}

1. Coughlin SS et al (2004) Diabetes mellitus as a predictor of cancer mortality in a large cohort of US adults. Am J Epidemiol 159:1160-1167

2. Larsson SC, Orsini N, Wolk A (2005) Diabetes mellitus and risk of colorectal cancer: a meta-analysis. J Natl Cancer Inst 97:16791687

3. Huxley R, Ansary-Moghaddam A, Berrington de Gonzalez A, Barzi F, Woodward M (2005) Type-II diabetes and pancreatic cancer: a meta-analysis of 36 studies. Br J Cancer 92:20762083

4. Larsson SC, Mantzoros CS, Wolk A (2007) Diabetes mellitus and risk of breast cancer: a meta-analysis. Int J Cancer 121:856862

5. Zendehdel K, Nyrén O, Östenson C-G, Adami H-O, Ekbom A, Ye W (2003) Cancer incidence in patients with type 1 diabetes mellitus: a population-based cohort study in Sweden. J Natl Cancer Inst 95:1797-1800

6. Giovannucci E, Michaud D (2007) The role of obesity and related metabolic disturbances in cancers of the colon, prostate and pancreas. Gastroenterology 132:2208-2225

7. Mulnier HE, Seaman HE, Raleigh VS, Soedamah-Muthu SS, Colhoun HM, Lawrenson RA (2006) Mortality in people with type 2 diabetes in the UK. Diabetic Med 23:516-521

8. Evans JMM, Donnelly LA, Emslie-Smith AM, Alessi DR, Morris $\mathrm{AD}$ (2005) Metformin and reduced risk of cancer in diabetic patients. BMJ 330:1304-1305

9. Bowker SL et al (2006) Increased cancer-related mortality for patients with type 2 diabetes who use sulfonylureas or insulin. Diabetes Care 29:254-258

10. Currie CJ, Poole CD, Gale EAM (2009) The influence of glucoselowering therapies on cancer risk in type 2 diabetes. Diabetologia doi:10.1007/s00125-009-1440-6

11. Zakikhani M, Dowling R, Fantus IG, Sonenberg N, Pollak M et al (2006) Metformin is an AMP kinase-dependent growth inhibitor for breast cancer cells. Cancer Res 66:10269-10273

12. Schimmack G, DeFronzo RA, Musi N (2006) AMP-activated protein kinase: role in metabolism and therapeutic implications. Diabetes Obes Metab 8:591-602

13. Sahra IB, Laurent K, Loubat A et al (2008) The antidiabetic drug metformin exerts an antitumour effect in vitro and in vivo through a decrease in cyclin D1 level. Oncogene 27:3576-3586

14. Pearce EL, Walsh MC, Cejas PJ et al (2009) Enhancing CD8 T cell memory by modulating fatty acid metabolism. Nature. doi:10.1038/nature08097

15. Yang Y-X, Hennessy S, Lewis JD (2004) Insulin therapy and colorectal cancer risk among type 2 diabetes mellitus patients. Gastroenterology 127:1044-1050 
16. Hsu IR, Kim SP, Kabir M, Bergman RN (2007) Metabolic syndrome, hyperinsulinemia, and cancer. Am J Clin Nutr 86:867S-871S

17. Holly JMP, Perks CM (2008) Cancer as an endocrine problem. Best Pract Res Clin Endocrinol Metab 22:539-550

18. Hanahan D, Weinberg RA (2000) The hallmarks of cancer. Cell 100:57-70

19. Samani AA, Yakar S, LeRoith D, Brodt P (2007) The role of the IGF system in cancer growth and metastasis: overview and recent insights. Endocr Rev 28:20-47

20. Kim JJ, Accili D (2002) Signalling through IGF-1 and insulin receptors: where is the specificity? Growth Horm IGF Res 12:84-90

21. Frasca F, Pandini G, Scalia P et al (1999) Insulin receptor isoform A, a newly recognised, high affinity insulin-like growth factor II receptor in fetal and cancer cells. Mol Cell Biol 19:3278-3288

22. Baserga R, Peruzzi F, Reiss K (2003) The IGF-1 receptor in cancer biology. Int J Cancer 107:873-877

23. Hemkens LG, Grouven U, Bender R et al (2009) Risk of malignancies in patients with diabetes treated with human insulin or insulin analogues: a cohort study. Diabetologia doi:10.1007/ s00125-009-1418-4

24. Jonasson JM, Ljung R, Talbäck M, Haglund B, Gudbjörnsdòttir S, Steineck G (2009) Insulin glargine use and short-term incidence of malignancies - a population-based follow-up study in Sweden. Diabetologia. doi:10.1007/s00125-009-1444-2

25. Colhoun HM, SDRN Epidemiology group (2009) Use of insulin glargine and cancer incidence in Scotland: a study from the Scottish Diabetes Research Network Epidemiology Group. Diabetologia. doi:10.1007/s00125-009-1453-1

26. http://www.fda.gov/Safety/MedWatch/SafetyInformation/SafetyRelatedDrugLabelingChanges/ucm122978.htm, accessed 15 June 1009

27. Mathieu C, Gale EAM (2008) Inhaled insulin: gone with the wind? Diabetologia $51: 1-5$

28. Berger M (2000) Safety of insulin glargine. Lancet 356:2013

29. Ebeling P, Tuominen JA, Koivisto VA (1996) Insulin analogues and carcinoma of the breast. Diabetologia 39:124-125

30. Hansen BF, Danielsen GM, Drejer K et al (1996) Sustained signalling from the insulin receptor after stimulation with insulin analogues exhibiting increased mitogenic potency. Biochem $\mathrm{J}$ 315:271-279

31. Slieker LJ, Brooke GS, DiMarchi RD et al (1997) Modifications in the B10 and B26-30 regions of the B chain of human insulin alter affinity for the human IGF-1 receptor more than for the insulin receptor. Diabetologia 40:S54-S61

32. Jorgensen L, Dideriksen L, Drejer K (1992) Carcinogenic effect of the human insulin analogue B10Asp in female rats. Diabetologia 35(Suppl 1):A3 (Abstract)

33. Kurtzhals P, Schäffer L, Sørensen A et al (2000) Correlations of receptor binding and metabolic and mitogenic potencies of insulin analogs designed for clinical use. Diabetes 49:999-1005

34. Erbel S, Büchler MW, Reers C et al (2008) Proliferation of colo357 pancreatic carcinoma cells and survival of patients with pancreatic carcinoma are not altered by insulin glargine. Diabetes Care 31:1105-1111

35. Weinstein D, Simon M, Yehezkel E, Laron Z, Werner H (2008) Insulin analogues display IGF-1-like mitogenic and anti-apoptotic activities in cultured cancer cells. Diabetes Metab Res Rev 25: 41-49

36. Shukla A, Grisouard J, Ehemann V, Hermani A, Enzmann H, Mayer D (2009) Analysis of signaling pathways related to cell proliferation stimulated by insulin analogs in human mammary epithelial cell lines. Endocr Relat Cancer 16:429-441

37. Kuerzel GU, Shukla U, Scholtz HE et al (2003) Biotransformation of insulin glargine after subcutaneous injection in healthy subjects. Curr Med Res Opin 19:34-40

38. Agin A, Jeandidier N, Gasser F, Grucker D, Sapin R (2007) Glargine blood biotransformation: in vitro appraisal with human insulin immunoassay. Diabetes Metab 33:205-212

39. Stammberger I, Bube A, Durchfeld-Meyer B, Donaubauer H, Troschau G (2002) Evaluation of the carcinogenic potential of insulin glargine (LANTUS) in rats and mice. Int J Toxicol 21:171-179

40. http://www.drugs.com/pro/lantus.html, accessed 15 June 2009

41. Rosenstock J, Fonseca V, McGill JB et al (2009) Similar progression of diabetic retinopathy with insulin glargine and neutral protamine Hagedorn (NPH) insulin in patients with type 2 diabetes: a long-term, randomised, open-label study. Diabetologia. doi:10.1007/s00125-009-1415-7

42. Schneider MB, Matsuzaki H, Haorah J et al (2001) Prevention of pancreatic cancer induction in hamsters by metformin. Gastroenterology 120:1263-1270

43. Matveyenko AV, Dry S, Cox HI et al (2009) Beneficial endocrine but adverse exocrine effects of sitagliptin in the HIP rat model of type 2 diabetes, interactions with metformin. Diabetes. doi: $10.2337 / \mathrm{db} 09-0058$

44. Rosenstock J, Schwartz SL, Clark CM Jr, Park GD, Donley DW, Edwards MB (2001) Basal insulin therapy in type 2 diabetes: 28-week comparison of insulin glargine (HOE 901) and NPH insulin. Diabetes Care 24:631-636

45. Zib I, Raskin P (2006) Novel insulin analogues and its mitogenic potential. Diabetes Obes Metab 8:611-620

46. Shaw LC, Grant MB (2004) Insulin like growth factor-1 and insulin-like growth factor binding proteins: their possible roles in both maintaining normal retinal vascular function and in promoting retinal pathology. Rev Endocr Metab Disord 5:199-207

47. Black N (1996) Why we need observational studies. BMJ 312:1215-1218

48. Papanikolaou PN, Christidi GD, Ioannidis JP (2006) Comparision of evidence on harms of medical interventions in randomized and non-randomized studies. CMAJ 174:635-641

49. Holleman F, Gale EAM (2007) Nice insulins, pity about the evidence. Diabetologia 50:1783-1790

50. Horvath K, Jeitler K, Berghold A, Ebrahim SH, Gratzer TW et al (2007) Long-acting insulin analogues vs NPH insulin (human isophane insulin) for type 2 diabetes mellitus. Cochrane Database Syst Rev issue 2:art. no. CD005613. doi:10.1002/14651858. CD005613.pub3.

51. Nathan DM, Buse JM, Davidson MB et al (2009) Medical management of hyperglycaemia in type 2 diabetes mellitus: a consensus algorithm for the initiation and adjustment of therapy. A consensus statement from the American Diabetes Association and the European Association for the Study of Diabetes. Diabetologia 52:17-30

52. Singh SR, Ahmad F, Lal A, Yu C, Bai Z, Bennett H (2009) Efficacy and safety of insulin analogues for the management of diabetes mellitus: a meta-analysis. CMAJ 180:385-397

53. Barone BB, Yeh H-C, Snyder CF et al (2008) Long-term all-cause mortality in cancer patients with pre-existing diabetes mellitus. A systematic review and meta-analysis. JAMA 300:2754-2764

54. Frayling TM, Colhoun H, Florez JC (2008) A genetic link between type 2 diabetes and prostate cancer. Diabetologia $51: 1757-1760$ 